\title{
A Serious Gaming Framework for Decision Support on Hydrological Hazards
}

Yusuf Sermet ${ }^{\text {a* }}$, Ibrahim Demir ab, and Marian Muste ${ }^{\text {b }}$,

${ }^{a}$ Department of Electrical and Computer Engineering, University of Iowa, Iowa City, IA, USA ${ }^{b}$

Department of Civil and Environmental Engineering, University of Iowa, Iowa City, IA, USA

\section{CONTACT}

Yusuf Sermet

msermet@uiowa.edu

Department of Electrical and Computer Engineering, University of Iowa

300 S. Riverside Dr., Iowa City, IA, 52246, USA

\section{ORCID}

Yusuf Sermet: http://orcid.org/0000-0003-1516-8335

Marian Muste: https://orcid.org/0000-0002-5975-462X

Ibrahim Demir: http://orcid.org/0000-0002-0461-1242

This manuscript has been submitted for publication in the Science of the Total Environment. Please note that the manuscript has yet to be formally accepted for publication. Subsequent versions of this manuscript may have slightly different content. If accepted, the final version of this manuscript will be available via the 'Peer-reviewed Publication DOI' link on the righthand side of this webpage. Please feel free to contact the corresponding author for feedback. 


\title{
A Serious Gaming Framework for Decision Support on Hydrological Hazards
}

\author{
Abstract \\ In this study, a web-based decision support tool (DST) was developed for hydrological multi-hazard \\ analysis while employing gamification techniques to introduce a competitive element. The serious \\ gaming environment provides functionalities for intuitive management, visualization, and analysis of \\ geospatial, hydrological, and economic data to help stakeholders in the decision-making process \\ regarding hydrological hazard preparedness and response. Major contributions of the presented DST \\ include involving the community in environmental decision making by reducing the technical complexity \\ required for analysis, increasing community awareness for the environmental and socio-economic \\ consequences of hydrological hazards, and allowing stakeholders to discover and discuss potential trade- \\ offs to hazardous scenarios considering the limitations in budget, regulations, and technicality. The paper \\ describes the software design approaches and system architecture applied for a modular, secure, and \\ scalable software as well as the framework's intuitive web-based user interfaces for real-time and \\ collaborative data analysis and damage assessment. Finally, a case study was conducted to demonstrate \\ the usability of DST in a formal setting and to measure user satisfaction with surveys.
}

Keywords: serious games, multi-hazard tournament, decision support systems, hydrological data management and visualization, web-based systems

\section{Introduction}

With increasing population and intensive changes of the agricultural and urban landscape, natural hazards are becoming a growing threat, coming in many forms, including floods, droughts, accelerated soil erosion, and water pollution from point and non-point sources [25],[35]. If the natural hazards impact the human environments, they often turn into disasters that can significantly disrupt society [21]. This definition of disaster conforms to the modern 
view of natural hazards as social events, where the people at risk are vulnerable to the effect of natural extreme events because of their social and environmental conditions [36]. Among allnatural hazards, floods are ranked as the most devastating socio-economic disasters in the U.S. [18] as well as in many other regions of the world [29].

Individually examined hazard risks may not always be linearly aggregated, and the hazards with causal relationships should be studied together to find the multi-hazard risks [10],[13]. Challenges to multi-hazard analysis include the difficulties in effectively communicating environmental data with easy-to-interpret visualizations [6],[32] due to extensive data collection and large-scale sensor networks [22]. Another challenge is to involve multiple actors in decisionmaking to obtain different perspectives in order to produce non-conflicting plans and policies [7]. Previous experience has shown that technical solutions do not always perform well to promote sustainability and adaptability strategies for practical situations, and thus recourse has been made to role-playing games where the stakeholders play an active role in the decisionmaking [14]. The co-production of decision-making through serious gaming with the involvement of local stakeholders in addition to management agencies has potential to improve the quality and efficiency of decision-making processes [2],[12]. By sharing the roles in formulating decisions, a game-like approach to the management of hazards can increase the viability of implementation plans considerably as the local stakeholders become the co-owners of the plan [31].

Prior examples of serious games in the disaster risk reduction and water management manifest the potential and benefits of gamified decision support systems [1],[17],[26]-[28],[34]. A major benefit of participatory serious gaming is the effective communication and education about complex phenomena to students, professionals, and officials as its examples are discussed in the 
literature [9],[11],[16],[23]. Various simulation games have been developed and tested for river basin management with an end goal to efficiently translate in practice scientifically-informed policy making [4],[20]. Wessels [33] presents a gaming environment for transboundary water management and cooperation and examines the role of players' identities, perceptions, and sociological backgrounds in decision-making. Marini et al. [15] discuss the best practices for developing serious games for integrated water resources management and emphasize the limitations of hydrological serious games that do not account for stakeholders' sociological and psychological perspectives.

A one-stop web-based Decision Support Tool (DST) for a multi-hazard (i.e. flood, drought, and water pollution) tournament was developed to support the delivery of the outcomes of a comprehensive hydrologic study to the targeted watershed community. The DST was designed to offer a serious-gaming environment that allows collaborative hazard mitigation planning through sound engagement of the community in decision-making using competitive gamification. This interactive environment entails a web application as a way to access, manage, visualize, and analyze geospatial, hydrological and economic parameters and data, and a relational database to efficiently store the information [5], its constraints, and semantic relationships [24]. The DST framework has been developed conforming to the Agile software development paradigm while employing industry conventions and design patterns for accuracy, maintainability, and performance.

The developed DST provides the opportunity for stakeholders (i.e. communities, residents, elected officials, and agency representatives of a watershed) to work together in order to address both the socio-economic and environmental impacts of hazards, in addition to reflecting their hydrologic perspectives. Thus, it allows communities to tackle the mitigation of water-related 
hazards by taking the social context into account instead of only the hydrological or technical perspective. The design of DST web platform is such that stakeholders are freed of the understanding of the technical complexity related to hydrological background when selecting and evaluating potential actions and focusing only on the decision-making aspects that they comprehend. This approach is a departure from the conventional decision-making whereby technical and managerial actors decide the solution to be implemented by the community. The presented DST adds a competitive element where teams independently decide between tradeoffs and create the best solution for a particular scenario set in the basin while dealing with each hazard-based scenario within the budgetary, temporal, spatial, technical, and regulatory constraints.

The remainder of this paper is organized as follows: Section 2 describes the details of the MultiHazard Tournament (MHT) as well as the data resources. Section 3 focuses on the software design and development process, implementation of the DST as a web-based platform and its system architecture, and user interaction and system functionalities. Section 4 discusses the case study. Section 5 presents the conclusions.

\section{The Multi-Hazard Tournament}

\subsection{Scope and Purpose}

This project follows the vision and conceptualization of a gamified multi-hazard mitigation approach (i.e. Multi-Hazard Tournament) presented by Carson et al. [3]. Previously, the MHT was executed with a fragmented approach and multiple tools requiring tedious data manipulations. The main purpose of the DST is to automate data flux within an intuitive, instructive, and collaborative web platform with geospatial visualization and analytics features 
included. The DST maximizes the tournament players' productivity by providing tools that automate most of the needed decision-making actions, including the cost and benefit calculations for various planning alternatives (e.g., introducing a freeboard policy, adding a detention basin). The DST has easy to use interfaces that enable stakeholder collaboration in evaluating and finally selecting appropriate watershed actions without getting into the details of the internal calculations.

Compared to previous MHT deliveries, the serious game presented in this paper includes new decision-making considerations that bring the planning process closer to reality and address new approaches to hazard mitigation. Specifically, the weather projections (i.e., wet or dry year sequence) is not pre-established in the formulation of the alternative scenarios, rather it is made known prior to the game though by a random scenario generator. This aspect is similar to the realistic planning situations whereby the mitigation trade-offs needs to account for the potential of flood and droughts that typically occur in an unknown sequence. The innovation regarding the hazard mitigation entails selection of solutions that are not only local but includes coordinated actions at the watershed scale. For this purpose, multiple "damage centers" are defined in floodprone areas of the watershed. Individual teams participating in the game select mitigation measures for both the community where they live as well as for township located upstream or downstream from their living spaces. This approach is more holistic by solving problems occurring in an interactive system that supposedly is more efficient than solving the problems locally. The DST uses the watershed of interest as the geographic context for decisions and provides maps with different layers (e.g. inundation maps) as well as various spatial data resources (e.g. critical infrastructure, land use) to facilitate stakeholder understanding. 


\subsection{Data Resources, Metrics, and Gameplay}

All data used for the tournament has been provided by the data and project partners in the form of large datasets provided by numerical simulations as well as spreadsheets and equations associated with the multi-criteria evaluation metrics (i.e., socio-economic damage data). Each damage center (DC) is provided with its corresponding parameters including current and future traditional critical infrastructure protection costs, infiltration and freeboard policy costs, cost of property buyout in the floodplain, anticipated growth factor, total monetary value of parcels in the flood plain, aggregated social vulnerability index, monetary values for wetland, riparian corridor, and riparian forest per acre per year (i.e. annual habitat value), and cost of reducing Escherichia coli (E. coli) and total suspended solids (TSS). In relation to the property buyout, the areas with a higher risk of flooding may be utilized for non-residential purposes. Independent from the DC, the cost of building parks and recreational facilities (e.g. trail, shelter, baseball and football fields, basketball court, picnic area, amphitheater, playground, and open area) is provided. For each alternative plan per DC, following parameters were pre-calculated by domain experts: the areas for wetland, riparian corridor, and riparian forest, TSS and E. coli loading, groundwater recharge volume, flood damages and impacted vulnerable population for 2, 10, and 100-year floods, and recreational facility benefits. Additionally, map layers for floodplain and habitat were produced, pre-processed, and stored as GeoJSON files for each alternative.

These information is presented to the players as appropriate for live feedback as the plans are selected and used in real-time to calculate six main scoring metrics assessing each plan's success. The scoring metrics are as follows: a) flood impacts (i.e. the cost of structural damage in the DC), b) groundwater recharge potential, c) protected habitat value, d) water quality (i.e. E. Coli and TSS loading in the stream), e) recreational benefits (i.e. increase in nearby property 
values), and f) impact to the vulnerable population (i.e. number of households with irreversible damages). The metrics are used to assign negative or positive scores to the team depending on whether the plan resulted in improvement or deterioration of infrastructure or threats to living matter (including the population exposed to hazard). The technical score is calculated by multiplying the normalized individual scores with weighting factors which are defined by the administrators. Additionally, the peer score is calculated by surveying the evaluations of the stakeholders for a team's plan in terms of appropriateness, viability, and consideration of impacts. The final score is the weighted sum of technical and peer scores and is the main factor for determining the winner.

The game consists of two consecutively executed rounds in which the players experiment with potential hazard mitigation strategies (i.e. plans). The main goal is to discover the most optimal mitigation strategy which maximizes environmental and humanitarian benefits while minimizing the tax-dollars spent, adding value to the communities, and keeping public contended with the taken actions. The players are tasked with evaluating trade-offs between actions while being provided with useful and timely information in a manner that is easy to interpret. The game is conducted and supervised by the tournament hosts who act as the DST administrators. The tournament rounds represent historical and future conditions regarding land use and development exposed to various weather patterns. At the end of each round, the teams present their plans to all game attendees for justification of their decisions and peer scoring. 


\section{DST Implementation}

\subsection{Software Design}

The requirements and expectations of the software are defined by The U.S. Army Corps of Engineers' (USACE) game design specifications. The DST is developed by abiding certain software development process rules to assure user satisfaction and platform soundness. When choosing the appropriate process, several factors have been taken into account. Domain experts in the environmental domain may not always speak the same technical language with the software developers. The developers (i.e., data scientists) and the domain specialists should work together to find efficient solutions to domain-focused problems using transparent, intelligible protocols that are understandable to all participants. Rather than specifying all software requirements at once at the beginning of the project, it is preferred to work with the developers to introduce software features as the product is being shaped incrementally. These conditions make it challenging to employ Big-Design-Up-Front software approach (e.g. Waterfall, Spiral) and suggest the use of Agile Software Development for the project.

Throughout the development, regular interaction between the domain specialists and the developers are assured with online and face-to-face meetings, weekly reports, and dry runs for each release after the minimum viable product. Both functional and non-functional requirements were gathered at the beginning of the project subject to modification. Behavior-driven development is employed to allow discussion and conversation between the stakeholders and the developers with the goal of specifying functional requirements in the way that they are consensual, complete, consistent, validated, and prioritized. Formally defining the behavior of a computational problem-solving system is often challenging for non-technical domain experts and 
professionals. Thus, user stories are used to elicit functional requirements of the system. They are described in a way that they are specific, measurable, and achievable in the aimed timeframe. Table 1 and Table 2 summarize the user stories for the actors of the system: the player and the admin, respectively. These user stories served as a basis for developing acceptance tests for system validation.

Table 1: Functional Requirement Elicitation: User Stories for Players

Table 2: Functional Requirement Elicitation: User Stories for Admins

Non-functional requirements of the application can be summarized as follows. The DST framework is designed to be generic; it should be independent of the location-specific parameters. For a replay, all the game components need to be able to reset via admin panel without requiring any modification to the code or the database. The DST should be hosted on a server to be accessed from the user's computer through a web browser without requiring any software installation. Certain procedures and visualizations need to be performed using the client's device to decrease the workload on the server. Calculations of the total scores for each team may not exceed 2 seconds, which is considered the acceptable wait time for the users, assuming the usage of prevalent personal computer hardware [30]. Different members of the same team need to be able to access to the DST simultaneously from different devices. Synchronization of different team members' actions must occur in real-time without requiring an explicit command or page reload. The system should work seamlessly and behave expectedly when accessed from a desktop computer or a mobile device. All of the functional and nonfunctional requirements have been taken into account when building the system's architecture and selecting the software frameworks. 


\subsection{System Architecture}

The presented serious-gaming framework has been implemented by using a 3-layer architecture which consists of Presentation, Logic, and Persistence layers. Figure 1 depicts the system architecture along with components and their interactions. As discussed in the literature extensively, the benefits of adopting 3-layer architecture range from increased development speed to better scalability due to its modularized nature. The presentation layer consists of a web server (i.e. Apache HTTP Server) to receive and respond to requests from the users by routing the queries to the appropriate handler (i.e. response generator) in the application. The main application is located on the server-side as part of the Logic Layer, as subsequently described in the following paragraph. The persistence (i.e. data) layer comprises the data storage and access system (i.e. PostgreSQL) to allow the Logic Layer to request and retrieve needed information via SQL queries. A relational database model has been designed using the metadata provided by the domain specialists as well as considering the application's requirements.

The main application is implemented using the Yii Framework based on PHP Hypertext Preprocessor (PHP). Yii framework has been chosen due to being open-source, its extensive security measures, automatic code generation, scalable and modularized architecture, and PHP's wide usage in server-side applications for mitigating future maintenance costs. Yii adopts the Model-View-Controller (MVC) design pattern which organizes the application code into 3 different layers to allow code reuse and parallel development. Models are focused on data retrieval and manipulation and are created for each entity in the database (e.g. damage centers, selections). No other component is allowed to communicate with the database directly. Views are responsible for the interface and interactions presented to the user and created for each page of the platform (e.g. Main Tournament, Scores Summary). Validation and execution of the user 
input and interactions are managed by the controllers in the context of taking actions utilizing the appropriate model(s) and view(s). The MVC pattern may seem similar to the 3-layer architecture. An important distinction that 3-layer architecture brings is that the tiers cannot communicate with each other arbitrarily. Instead, the flow of information will occur linearly unlike the Model-View-Controller (MVC) architectural pattern where the communication between blocks can happen in a triangular manner.

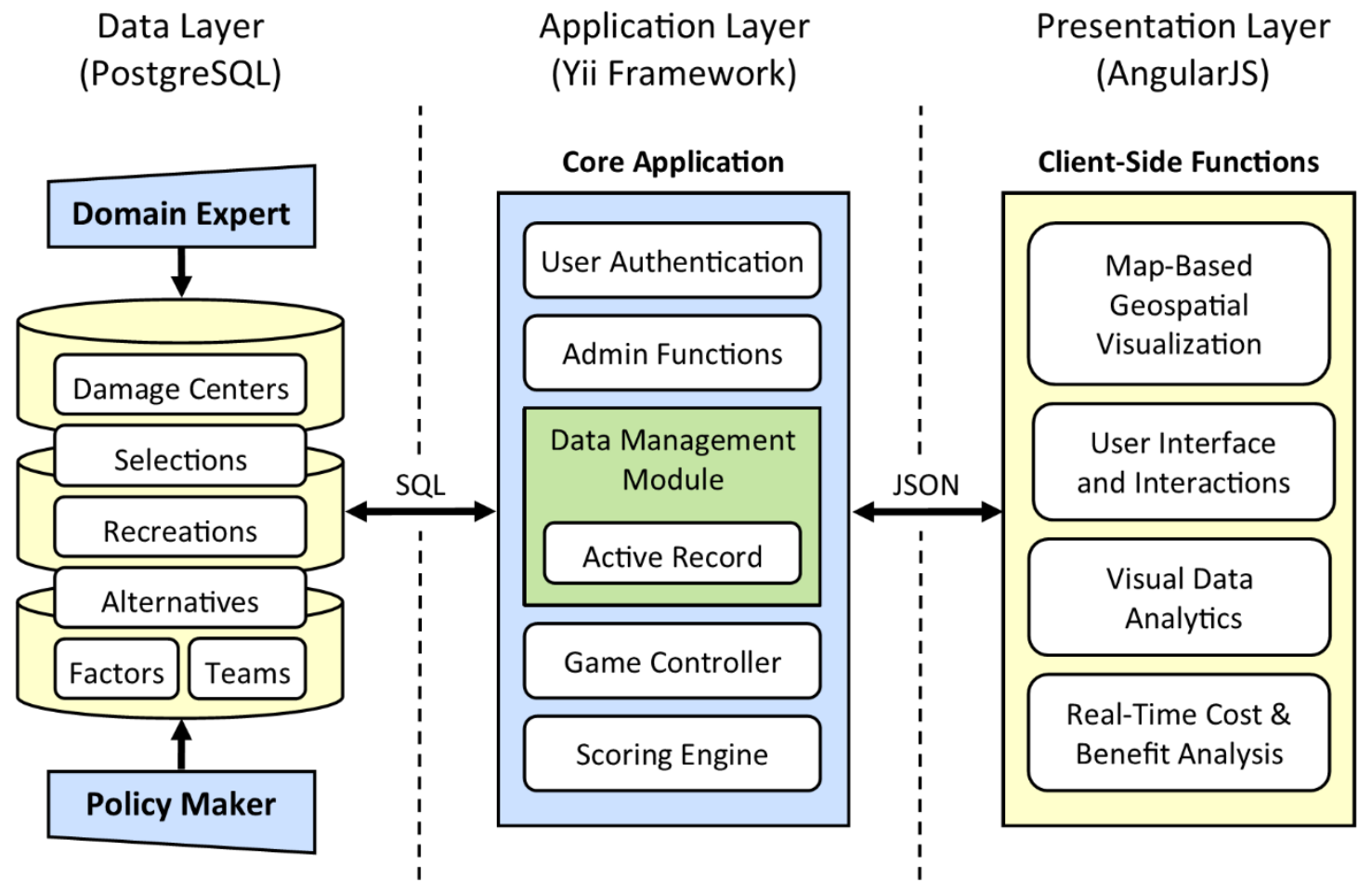

Figure 1: System Architecture

The core application consists of several semantic components. User Authentication Module is responsible for the registration, login, and session management for both the admins and tournament players. Admin Functions Module implements all the capabilities to manage, visualize, analyze, and reset the tournament, scores, game parameters, and teams. Game Controller solely focuses on the gameplay and rules, whereas the Scoring Engine's scope extends 
to the efficient and real-time calculation of the scores while implementing smart caching mechanisms and avoiding memory leaks. Data Management Module provides the libraries and workflow for communicating with the database using Active Record pattern to simplify the CRUD (i.e. create, read, update, delete) operations. Figure 2 shows the relational database model that the Data Management Module is designed upon.

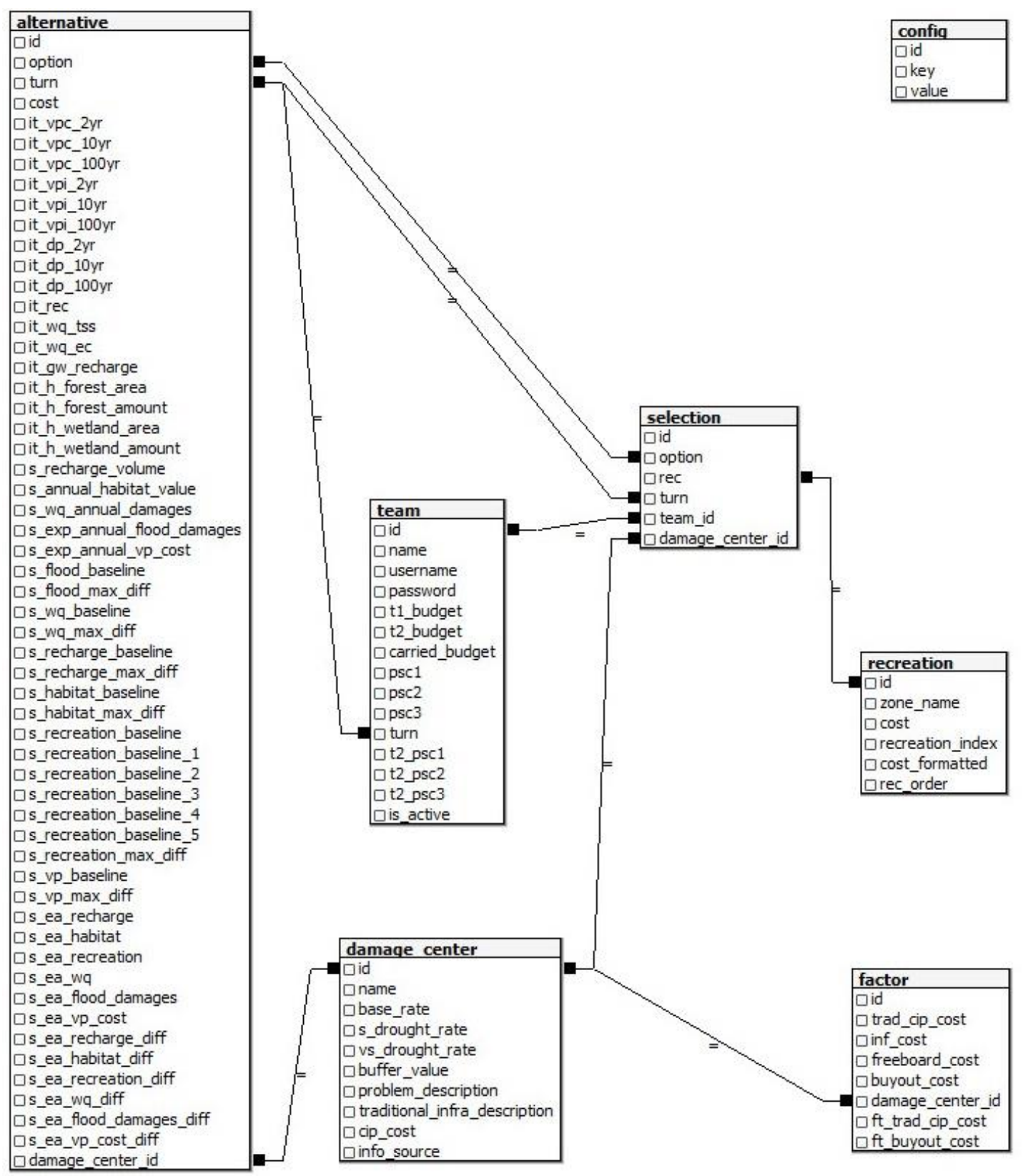

Figure 2: Database Schema for the Multi-Hazard Tournament 
A separate framework, namely AngularJS, has been adopted for implementing client-side functionalities. This adoption allows the application to cleanly decouple the server-side application logic from the user interface and interactions, which simplifies development and maintenance. Similar to Yii Framework, AngularJS also follows the MVC architectural pattern, which assures consistency throughout the whole application and decreases the variety of skills needed by the developers, which allows the developers to change roles between the frontend and backend tasks when necessary. For consistency, lightweight, and responsive styling, Bootstrap CSS Framework is used which also helps to make the platform compatible for multiple screen sizes. The framework and language preferences described in this paper do not have any intrinsic dependencies to the presented serious gaming platform, and they are simply design choices for which one of the factors is being open-source. Thus, the multi-hazard tournament can be implemented in any web application development framework (e.g. Django with Python, Ruby on Rails).

\subsection{User Interaction and System Functionalities}

The platform has two aspects of abstraction customized for the type of users involved: players (i.e. any stakeholder including public, state and federal agencies, local/municipal governments, and researchers) and administrators (e.g. domain specialists and managers). These two aspects are assembled into two semantic blocks: Tournament Module and Administration Module. A critical feature of the platform is that the simultaneous access by multiple users is supported with precautions to prevent any race condition [19] in place. All of the actions taken by the players during the game are recorded in their corresponding states in the database in real-time. 
The Tournament Module consists of a one-page interactive environment (Figure 3) for the entirety of the serious game and the summary page for team scores and selections (Figure 4).

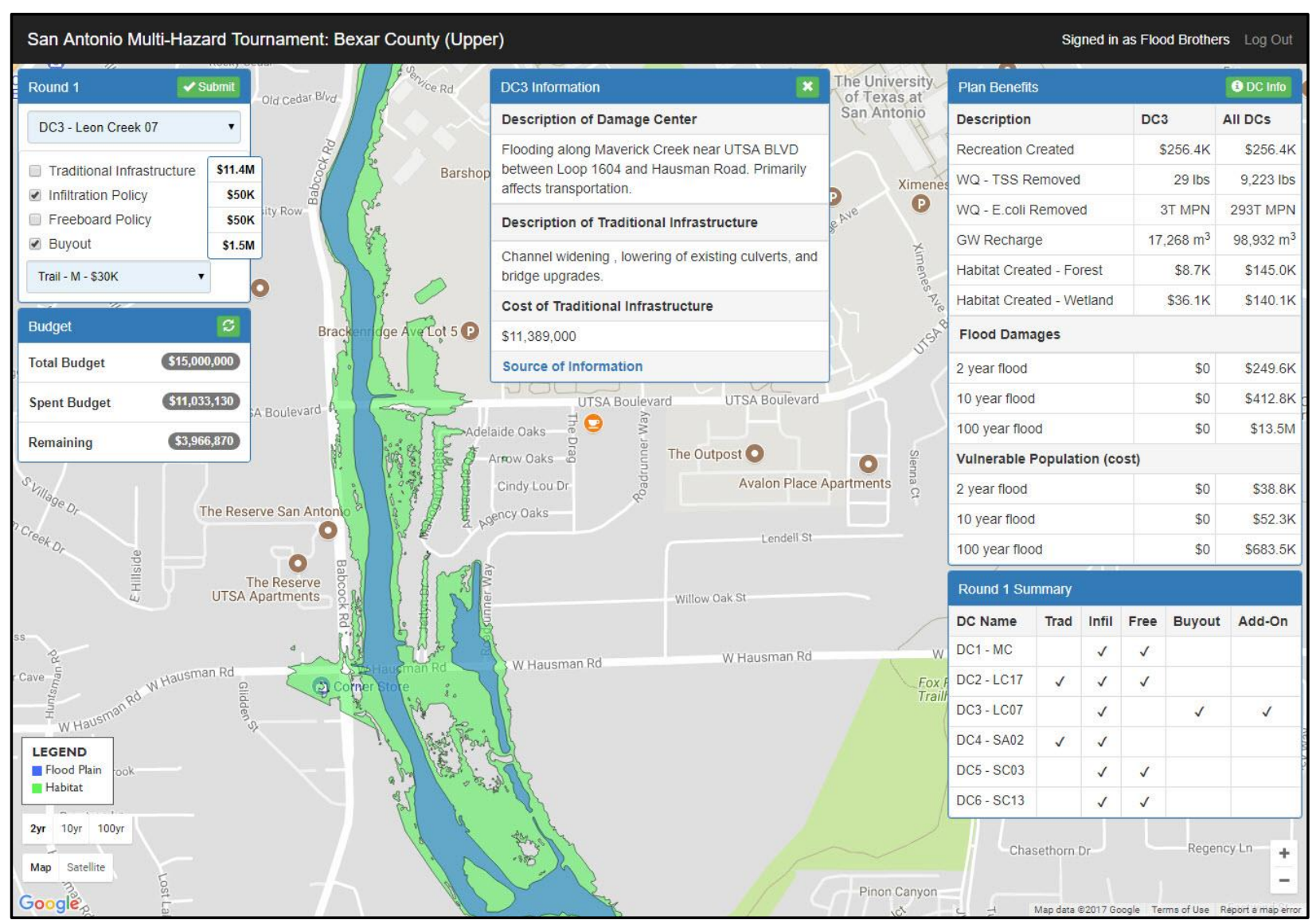

Figure 3: Gameplay - Multi-Hazard Tournament Main Application Page

Upon the game's initialization, several panels are used to effectively present the information player needs. Damage centers can be navigated to discover the DCs as well as its nearby topography and city plans from the map. For each DC, the location and traditional infrastructures' description and cost are given as a panel. Sources of information are available for each DC should further investigation by the players is desired. The financial cost of each precautionary action and their scopes are presented as checkboxes for players to experiment with while keeping track of the assigned budget. Upon each action taken by the player, the benefits 
and consequences of their plan are recalculated and reflected in the form of a table to make financial, environmental, and humanitarian impact clear for the active DC and for overall outlook. An additional reason that makes the overall outlook critical after each singular action is that certain DCs are connected to each other regarding their relationship in terms of the flood plain and geographical conditions. Connected DCs are processed in the server-side to perform the auxiliary and indirect effects to corresponding DCs and reflect back to the player in real-time for evaluation.

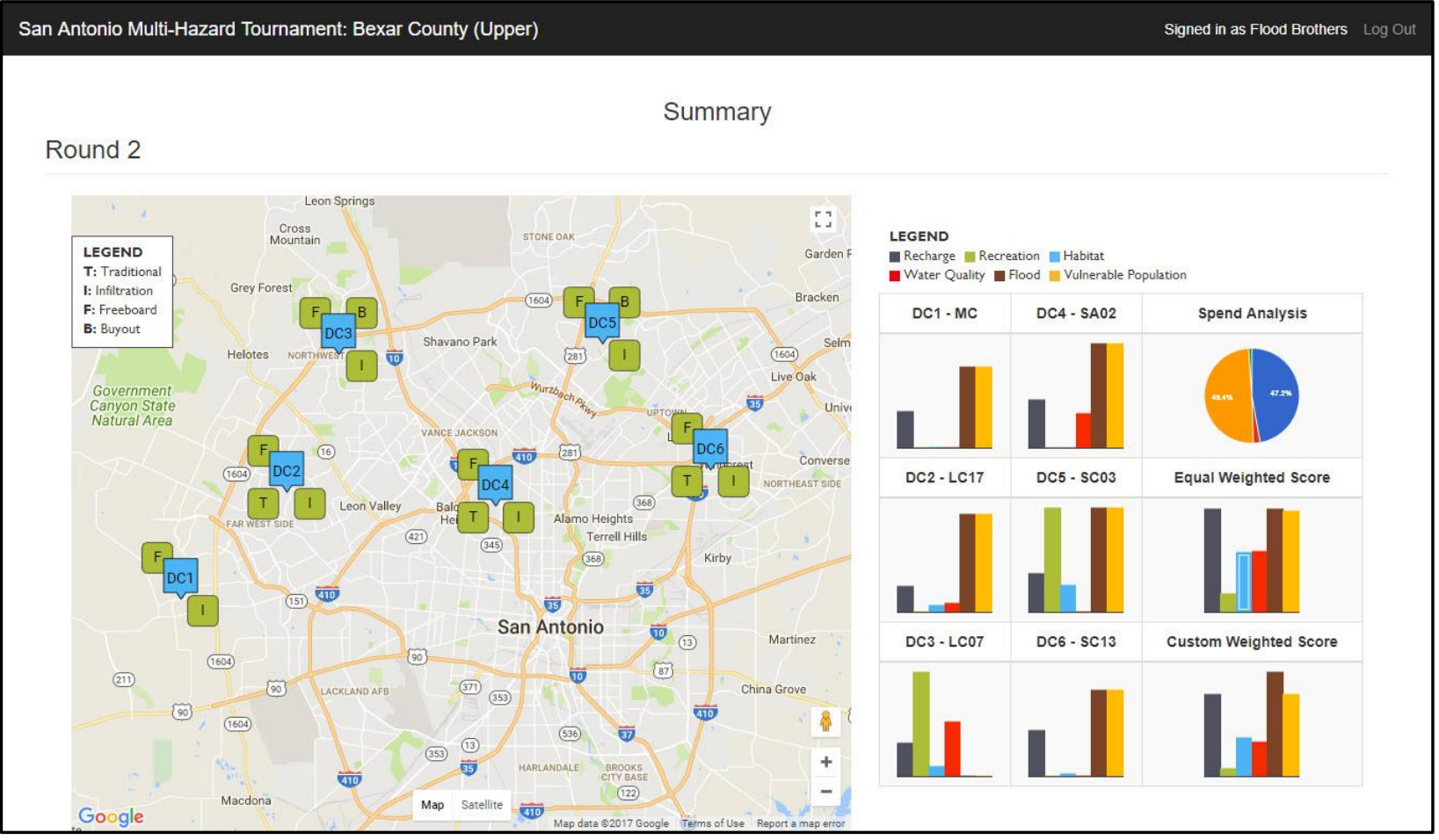

Figure 4: Tournament Module - End-of-Round Summary Page

The players are able to interact with the map (i.e. street or satellite views) with visualization of the eco-habitat and flood plain maps for each DC using 2 year, 10 year, and 100 year return periods. The data layers for eco-habitat and flood plain maps are loaded dynamically as polygons using Google Maps Platform's GeoJSON data loading library. Due to their complexity, the data 
layers were conditioned to reduce noise and for smooth representation and prompt loading. Upon submission of the selections, the summary page is displayed to provide the summary of the team's selections per damage center (DC) on an interactive map along with calculated impacts per DC, spend analysis, and equal and custom-weighted scores in the form of interactive bar and pie charts. This page is displayed after each round of the game to give the team the opportunity to review, discuss, and take lessons from their actions. Figure 5 shows the sequence diagram describing main flow of actions taken by a player in a game round.

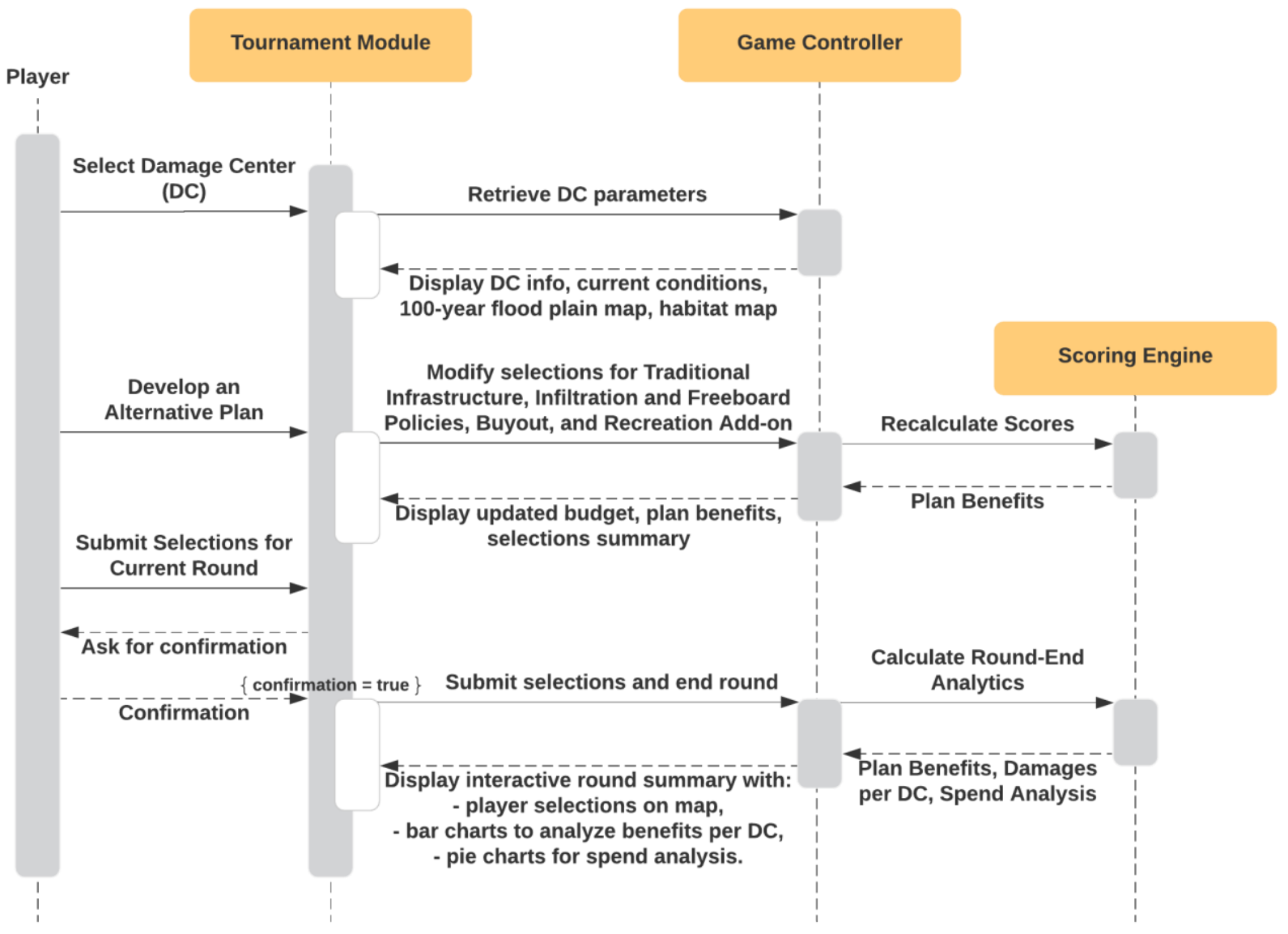

Figure 5: System-level sequence diagram representing the flow of actions from a player's perspective. Player authentication is omitted (i.e. player is assumed to be logged in.). 
Unlike the Tournament Module, the Administration Module consists of several pages and is designed for use by tournament referees and managers. The dashboard provides the real-time status of each team during the game and allows the admin to end a round or start a new round once all the teams have submitted their selections. Teams can only be created and modified through the Team Management page by the admins, who are responsible for assigning credentials, budgets, bonds, and peer scores, for full accountability. Weighting factors are determined for each plan benefit (i.e. Flood, Water Quality, Groundwater Recharge, Habitat, Recreation, and Vulnerable Population) to quantify the weight of each benefit in the final technical score. The sequence of extreme event (i.e. droughts and floods) occurrences and return periods are specified to estimate the long-term benefits in preventing infrastructural damages of groundwater recharge and housing exposed to flooding and for the protection of vulnerable population. Both factors are defined using web forms with responsive rule validation.

There are three interfaces for analysis and visualization of results, each presenting the scores at a different granularity. The first one presents the selections and benefit-specific scores for each DC per team per round with the company of equal and custom-weighted technical and peer scores. The second one interactively visualizes the summary of each team's plan benefits for weighted and unweighted estimated annual impact, actual impact, and peer scores per round (Figure 6). The interactive charts used in this project have been implemented using Google Chart JavaScript library for accuracy, consistency, and performance. The last page simply provides the final results of the tournament with scores for each team sortable by each metric, thus outlining the winner. Figure 7 provides the sequence diagram outlining the necessary steps for admin to initialize or reset the DST to make it ready for a new tournament. 


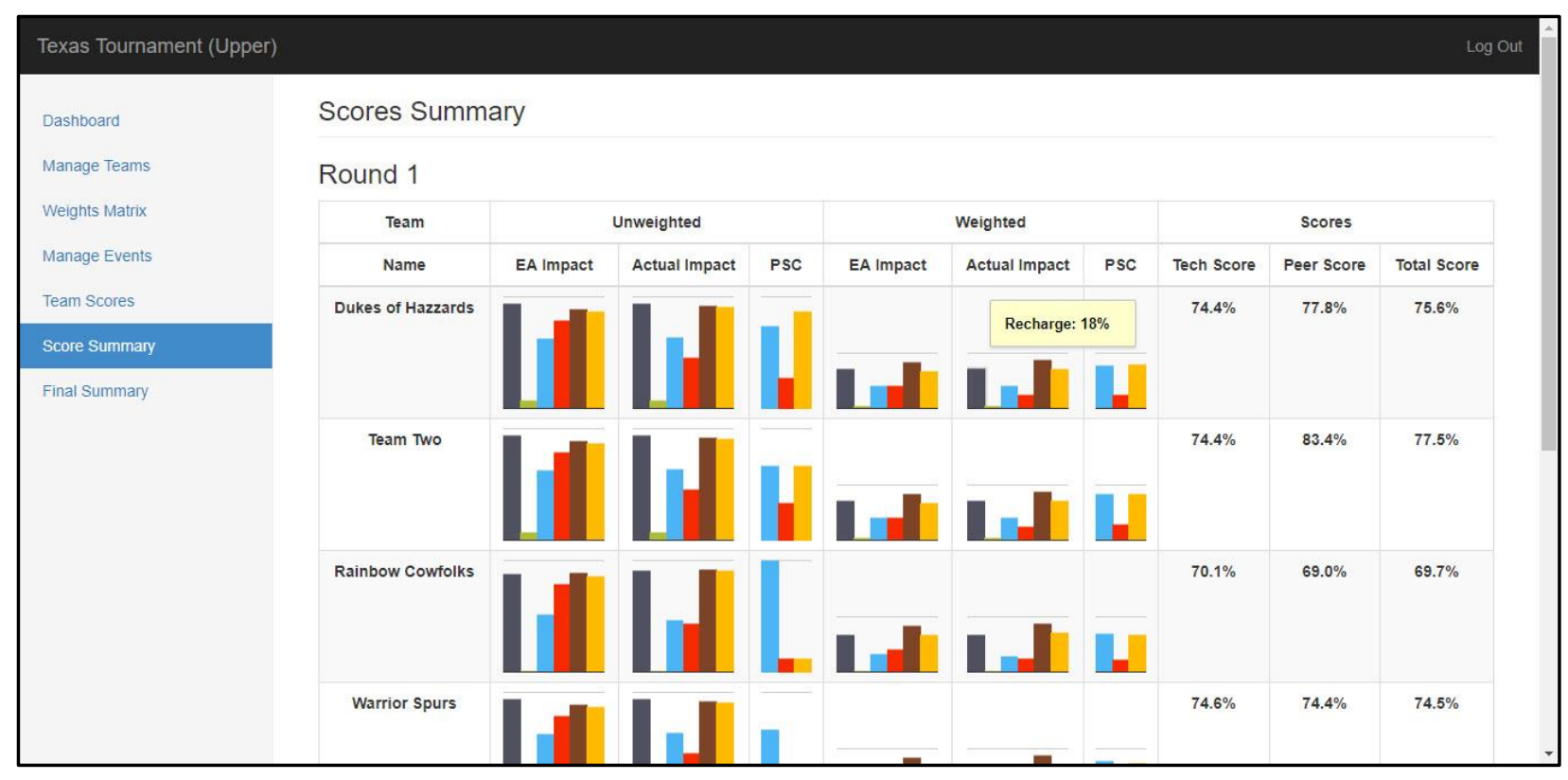

Figure 6: Administration Module - Interactive Visualization of Scores Summary

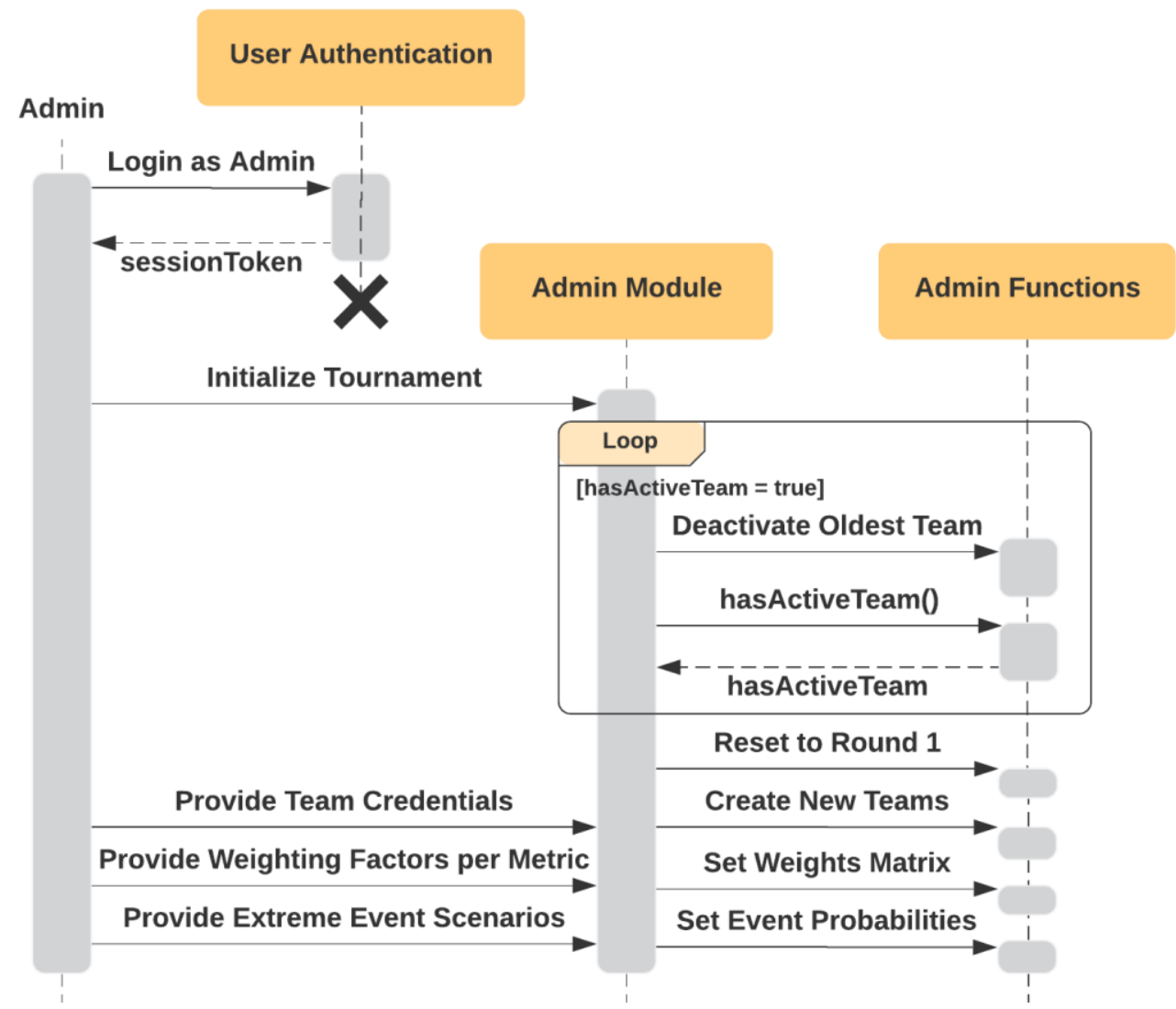

Figure 7: System-level sequence diagram describing the initialization of the DST. 


\section{Case Study}

The case study for testing the DST was conducted for two basins spanning the Bexar, Wilson, Karnes, and Goliad Counties in Texas (USA) in July 2017 (Figure 8). Due to their vulnerability to recurrent multi-hazard damages, these counties were found suitable for evaluating the presented MHT framework. Sixty individuals from a variety of backgrounds (e.g. agriculture, regional planning, hazard management, local government emergency units, federal agency, academia, tourism) participated in the game. One day prior to the event, the organizers distributed the participants the playbook detailing the gameplay. Early on the game day, participants were introduced to the purpose and scope of the game and they received details of the gameplay and user interfaces. Before and after the game, the participants completed surveys designed to assess their backgrounds, understanding of the multi-hazard risks and mitigation strategies, and their degree of satisfaction with the MHT utility. The surveys included openended questions which allowed the players to freely articulate their thoughts. A 5-point Likert Scale style ( $1=$ Completely Disagree, $2=$ Somewhat Disagree, $3=$ Neutral, $4=$ Somewhat Agree, $5=$ Completely Agree) questionnaire was used to rate their agreement on items measuring understanding and satisfaction.

According to the results of the questionnaires, participants obtained higher mean scores on items measuring understanding of mitigation and management approaches for hydrological hazards as well as disaster risks. In the post-event survey, the players described detailed plans and suggested additions for composing real-life scenarios. The substantial answers provided through the survey by the players indicate that they understood and were engaged in the game delivery and they cared about the issues detailing the risks that the disasters pose to their communities and shared watershed. The participants' agreement on questionnaire items such as "The decision support 
tool was useful during the Multi-Hazard Tournament", "Length and pace of the tournament were appropriate", and "Hazard scenarios provided a realistic context for decision making" were highly satisfactory $(M=4.55,4.26$, and 4.22 , respectively). $92 \%$ of the respondents stated that they were more comfortable using watershed planning information to make decisions and plan for the future after participating in the DST-based tournament. It can be concluded from the survey that the DST facilitated an effective, efficient, instructive, and pleasant MHT execution.
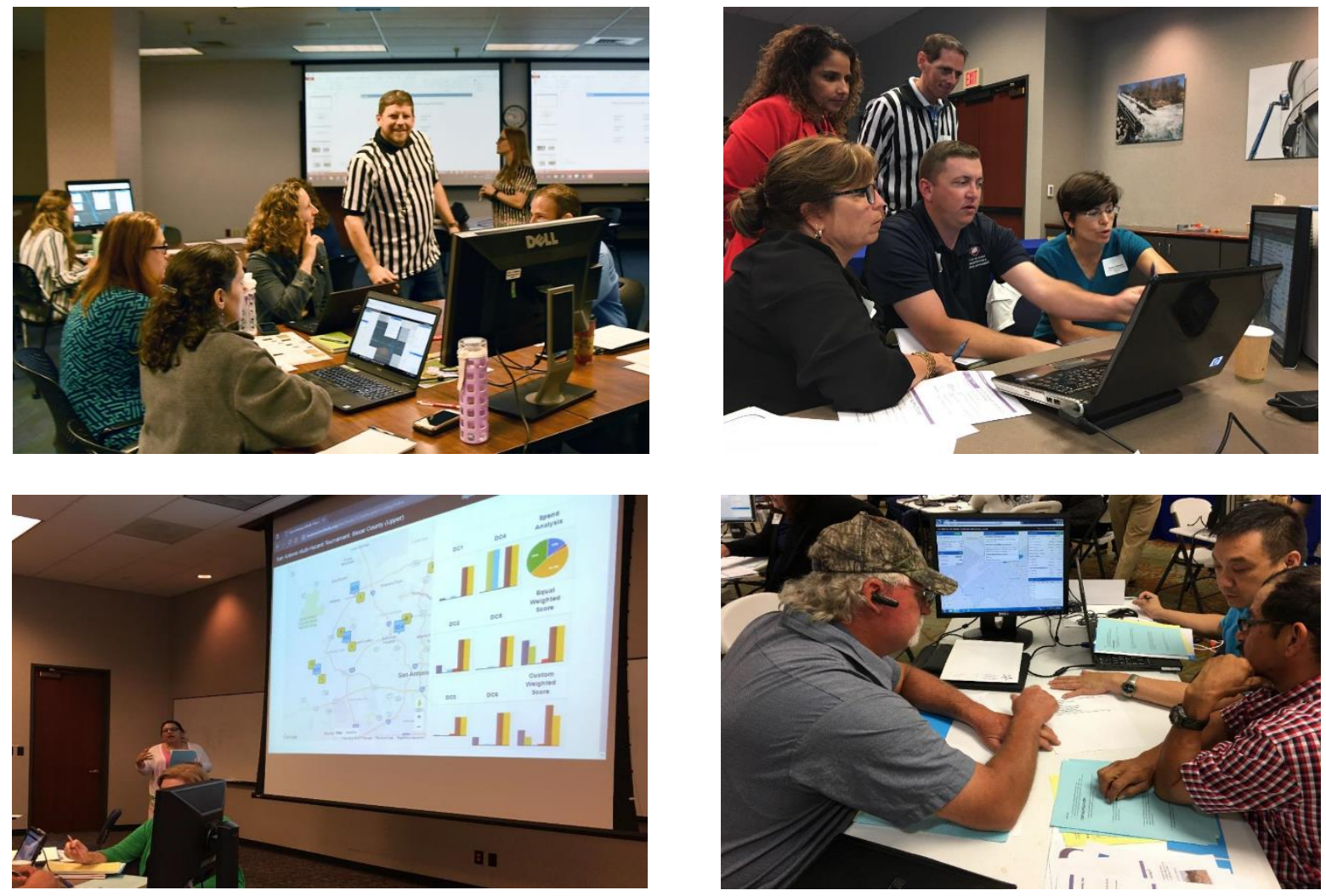

Figure 8: Snapshots from the Case Study - Bexar County (San Antonio, TX)

\section{Conclusions}

This paper presents a web-based Multi-Hazard Tournament framework including the hydrological background, the approach used for organizing the gaming approach, the development workflow, and the functionality as a data analytics tool. The site-delivery of the 
DST in actual serious gaming situations illustrates that such contemporary tools can empower stakeholders to take part in the decision-making process and co-produce precautionary measures for multiple natural hazard mitigation. More importantly, the involvement of the actual users in the decision-making uniquely facilitated by the DST facilitates increased awareness of the socioeconomic and environmental consequences of hydrological hazards and gives to the community a sense of ownership of the mitigation plans. Despite of the high-technical complexity of the DST architecture, components, and of the multi-disciplinary technical background involved in the hazard simulation and assessment, the DST interfaces allow seamless, effective, and realtime communication of the information to a wide range of audiences while saving time in analysis. In short, the DST suppress the technical and computer domain complexities involved in the hazard mitigation planning, making possible for the stakeholders to focus on important issues that they understand and enabling them to strategically decide on the fate of their watersheds with due considerations to the local social and economic dynamics.

\section{Acknowledgement}

This project is based upon work funded by the US Army Corps of Engineers and studied in collaboration with The Institute for Water Resources (Fort Worth District) and the San Antonio River Authority. Special thanks to our agency colleagues Aarin Teague, Andrea Carson, Harvey Hill, and Rolf Olsen. Haowen Xu and Haider Hameed, graduate students with IIHRHydroscience and Engineering, provided valuable input and assistance towards the development of the presented framework. 


\section{References}

[1] Aubert, A.H., Bauer, R. and Lienert, J., 2018. A review of water-related serious games to specify use in environmental Multi-Criteria Decision Analysis. Environmental Modelling \& Software, 105, pp.64-78.

[2] Cardwell, H., Langsdale, S., and Stephenson, K., 2009. A shared vision planning primer: How to incorporate computer aided dispute resolution in water resources planning. US Army Corps of Engineers, Institute for Water Resources and Virginia Polytechnic Institute. Alexandria, VA.

[3] Carson, A., et al., 2018. Serious gaming for participatory planning of multi-hazard mitigation. International journal of river basin management. 16.3: 379-391.

[4] Craven, J., et al., 2017. Development and testing of a river basin management simulation game for integrated management of the Magdalena-Cauca river basin. Environmental modelling \& software, 90, pp.78-88.

[5] Demir, I. and Szczepanek, R., 2017. Optimization of river network representation data models for web-based systems. Earth and Space Science, 4(6), pp.336-347.

[6] Demir, I., et al., 2018. FLOODSS: Iowa flood information system as a generalized flood cyberinfrastructure. International journal of river basin management, 16(3), pp.393-400.

[7] den Haan, R.J., et al., 2019. Understanding actor perspectives regarding challenges for integrated river basin management. International journal of river basin management, 17(2), pp.229-242.

[8] Department of Homeland Security (DHS) (2018). D. Alexander and D. Cotter "Flood Apex Research Program - Findings and Progress, Science \& Technology, U.S. Department of Homeland Security, presentation at the Floodplain Management Association Conference, Reno, NV, on 09/05/2018 (Available on line at: https://www.asfpmfoundation.org/aceimages/LALspeaker_series)

[9] Flood, S., et al. "Adaptive and interactive climate futures: systematic review of 'serious games' for engagement and decision-making." Environmental Research Letters 13.6 (2018): 063005 .

[10] Gallina, V., et al. "A review of multi-risk methodologies for natural hazards: Consequences and challenges for a climate change impact assessment." Journal of environmental management 168 (2016): 123-132. 
[11] Hoekstra, A.Y., 2012. Computer-supported games and role plays in teaching water management. Hydrology and earth system sciences, 16(8), pp.2985-2994.

[12] Jean, S., et al. "Serious games as a catalyst for boundary crossing, collaboration and knowledge co-creation in a watershed governance context." Journal of environmental management 223 (2018): 1010-1022.

[13] Kappes, M.S., et al., 2012. Challenges of analyzing multi-hazard risk: a review. Natural hazards, 64(2), pp.1925-1958.

[14] Komendantova, N., et al., 2014. Multi-hazard and multi-risk decision-support tools as a part of participatory risk governance: Feedback from civil protection stakeholders. International Journal of disaster risk reduction, 8: 50-67.

[15] Marini, D., et al., 2018. Socio-psychological perspectives on the potential for serious games to promote transcendental values in IWRM decision-making. Water, 10(8), p.1097.

[16] Markwart, H., et al. "Warning messages to modify safety behavior during crisis situations: a virtual reality study." International journal of disaster risk reduction 38 (2019): 101235.

[17] Muste, M., et al, 2017, September. Community engagement in water resources planning using serious gaming. In 2017 13th IEEE International Conference on Intelligent Computer Communication and Processing (ICCP) (pp. 445-451). IEEE.

[18] National Research Council. 2012. Disaster Resilience: A National Imperative. Washington, DC: The National Academies Press. https://doi.org/10.17226/13457.

[19] Netzer, R.H. and Miller, B.P., 1992. What are race conditions?: Some issues and formalizations. ACM Letters on Programming Languages and Systems (LOPLAS), 1(1), pp.74-88.

[20] Onencan, A., Enserink, B. and Van de Walle, B., 2018. A Study of Trust and Cooperation in the Nzoia River Basin Using a Water Policy Game. Sustainability, 10(12), p.4678.

[21] Santos, P.P., et al. "A comprehensive approach to understanding flood risk drivers at the municipal level." Journal of Environmental Management 260 (2020): 110127.

[22] Sermet, Y., et al., 2019. "Crowdsourced approaches for stage measurements at ungauged locations using smartphones." Hydrological Sciences Journal, 1-10.

[23] Sermet, Y. and Demir, I., 2019. Flood action VR: a virtual reality framework for disaster awareness and emergency response training. In ACM SIGGRAPH 2019 Posters (pp. 1-2). 
[24] Sermet, Y. and Demir, I., 2019. Towards an information centric flood ontology for information management and communication. Earth Science Informatics, 12(4), pp.541-551.

[25] Sit, M.A., Koylu, C. and Demir, I., 2019. Identifying disaster-related tweets and their semantic, spatial and temporal context using deep learning, natural language processing and spatial analysis: a case study of Hurricane Irma. International Journal of Digital Earth, 12(11), pp.1205-1229.

[26] Solinska-Nowak, Aleksandra, et al. "An overview of serious games for disaster risk management-Prospects and limitations for informing actions to arrest increasing risk." International journal of disaster risk reduction 31 (2018): 1013-1029.

[27] Tanwattana, P. and Toyoda, Y., 2018. Contributions of gaming simulation in building community-based disaster risk management applying Japanese case to flood prone communities in Thailand upstream area. International journal of disaster risk reduction, 27, pp.199-213.

[28] Tsai, M., et al. "Exploring the effects of a serious game-based learning package for disaster prevention education: The case of Battle of Flooding Protection." International Journal of Disaster Risk Reduction 43 (2020): 101393.

[29] UNISDR, U., 2015, March. Sendai framework for disaster risk reduction 2015-2030. In Proceedings of the 3rd United Nations World Conference on DRR, Sendai, Japan (pp. 1418).

[30] University of Iowa Information Technology Services, 2019. Suggested Computer Configurations for Students. [online]. Available from: https://its.uiowa.edu/support/article/100895 [Accessed 15 May 2019].

[31] Voinov, A., et al., 2016. Modelling with Stakeholders - Next Generation, Environmental Modelling \& Software. 77, 196-220.

[32] Weber, L.J., et al., 2018. The Iowa Watersheds Project: Iowa's prototype for engaging communities and professionals in watershed hazard mitigation. International journal of river basin management, 16(3), pp.315-328.

[33] Wessels, J.I., 2016. "Playing the game", identity and perception-of-the-other in water cooperation in the Jordan River Basin. Hydrological Sciences Journal, 61(7), pp.1323-1337. 
[34] Xu, H., et al. "A web-based decision support system for collaborative mitigation of multiple water-related hazards using serious gaming." Journal of Environmental Management 255 (2020): 109887.

[35] Yildirim, E. and Demir, I., 2019. An integrated web framework for HAZUS-MH flood loss estimation analysis. Natural Hazards, 99(1), pp.275-286.

[36] Zhou, Lei, et al. "Emergency decision making for natural disasters: An overview." International journal of disaster risk reduction 27 (2018): 567-576. 


\section{Figures}

Figure 1: System Architecture ......................................................................................... 12

Figure 2: Database Schema for the Multi-Hazard Tournament ........................................... 13

Figure 3: Gameplay - Multi-Hazard Tournament Main Page................................................. 15

Figure 4: Tournament Module - End-of-Round Summary Page ........................................... 16

Figure 5: System-level sequence diagram representing the flow of actions from a player's perspective. Player authentication is omitted (i.e. player is assumed to be logged in.).............. 17

Figure 6: Administration Module - Interactive Visualization of Scores Summary .................... 19

Figure 7: System-level sequence diagram describing the initialization of the DST................. 19

Figure 8: Snapshots from the Case Study - Bexar County (San Antonio, TX) ........................ 21 
Table 1: Functional Requirement Elicitation: User Stories for Players

\begin{tabular}{|c|c|c|}
\hline User Story & Scenario & Step-by-Step Description \\
\hline $\begin{array}{l}\text { Select } \\
\text { Alternative }\end{array}$ & $\begin{array}{l}\text { Player selects } \\
\text { alternatives for } \\
\text { the desired } \\
\text { damage center. }\end{array}$ & $\begin{array}{l}\text { 1. Player selects a damage center (DC) from the drop-down list. } \\
\text { 2. System updates the current DC to the selected one, shows the } \\
\text { DC information panel, updates Plan Benefits, and loads } \\
\text { floodplain and habitat GIS files onto the map. } \\
\text { 3. Player selects/deselects alternatives including Traditional } \\
\text { Infrastructure, Infiltration Policy, Freeboard Policy, Buyout, } \\
\text { and Recreation Add-on. } \\
\text { 3.1. Player may check the detailed descriptions for benefits by } \\
\text { hovering. } \\
\text { 4. System updates budget, plan benefits, and round summary } \\
\text { table. }\end{array}$ \\
\hline $\begin{array}{l}\text { Update } \\
\text { Floodplain }\end{array}$ & $\begin{array}{l}\text { Player selects } \\
\text { return period } \\
\text { for flood plain. }\end{array}$ & $\begin{array}{l}\text { 1. Player selects a damage center (DC) from the drop-down list. } \\
\text { 2. System loads the flood plain and habitat GIS files for } 100 \\
\text { year return period. } \\
\text { 3. Player selects a return period using the buttons placed left } \\
\text { bottom corner of the page. } \\
\text { 4. System updates the floodplain according to the selected } \\
\text { return period. }\end{array}$ \\
\hline $\begin{array}{l}\text { Submit } \\
\text { Round }\end{array}$ & $\begin{array}{l}\text { Player submits } \\
\text { the selections } \\
\text { for the current } \\
\text { round. }\end{array}$ & $\begin{array}{l}\text { 1. Player clicks on the submit button placed on the left upper } \\
\text { corner of the screen. } \\
\text { 2. System presents a warning dialog for player to confirm } \\
\text { irreversible action. } \\
\text { 3. Player clicks on confirm button to confirm. } \\
\text { 4. System processes the selections and presents a visual } \\
\text { summary page for player to see the summary of the selections } \\
\text { on the map, bar charts stating the scores for each DC, and pie } \\
\text { chart stating the spend analysis. }\end{array}$ \\
\hline
\end{tabular}


Table 2: Functional Requirement Elicitation: User Stories for Admins

\begin{tabular}{|c|c|c|}
\hline $\begin{array}{l}\text { User } \\
\text { Story }\end{array}$ & Scenario & Step-by-Step Description \\
\hline $\begin{array}{l}\text { Manage } \\
\text { Teams }\end{array}$ & $\begin{array}{l}\text { Admin creates, } \\
\text { updates, } \\
\text { deletes teams. }\end{array}$ & $\begin{array}{l}\text { 1. Admin clicks on Manage Teams tab on the menu. } \\
\text { 2. System presents associated details for every team as a table, } \\
\text { along with options to create, update, reset, and delete any team. } \\
\text { 3a. Admin adds new team } \\
\text { 3a.1 Admin fills the fields for name, username, and password, } \\
\text { then clicks submit. } \\
\text { 3a.2. Return user to step } 2 \text {. } \\
\text { 3b. Admin updates a team } \\
\text { 3b.1. Admin updates the desired fields and clicks submit. } \\
\text { 3b.2. Return user to Step } 2 \\
\text { 3c. Admin resets/deletes a team } \\
\text { 3c. } 1 \text { System presents a warning dialog for admin to confirm } \\
\text { irreversible action. } \\
\text { 3c. } 2 \text {. Admin clicks on confirm button to confirm. } \\
\text { 3c. } \text {. Return user to step } 2 \text {. }\end{array}$ \\
\hline $\begin{array}{l}\text { Update } \\
\text { Weights }\end{array}$ & $\begin{array}{l}\text { Admin updates } \\
\text { the weights } \\
\text { matrix for the } \\
\text { active } \\
\text { tournament. }\end{array}$ & $\begin{array}{l}\text { 1. Admin clicks on Weights Matrix tab on the menu. } \\
\text { 2. System shows a page with editable fields. } \\
\text { 3. Admin updates the desired fields and clicks save button. } \\
\text { 4. System presents a success message. }\end{array}$ \\
\hline $\begin{array}{l}\text { Manage } \\
\text { Events }\end{array}$ & $\begin{array}{l}\text { Admin updates } \\
\text { event periods } \\
\text { for each round. }\end{array}$ & $\begin{array}{l}\text { 1. Admin clicks on Manage Events tab on the menu. } \\
\text { 2. System shows a page with editable fields. } \\
\text { 3. Admin updates the desired fields and clicks save button. } \\
\text { 4. System presents a success message. }\end{array}$ \\
\hline $\begin{array}{l}\text { Display } \\
\text { Team } \\
\text { Scores }\end{array}$ & $\begin{array}{l}\text { Admin views } \\
\text { detailed team } \\
\text { scores. }\end{array}$ & $\begin{array}{l}\text { 1. Admin clicks on Team Scores tab on the menu. } \\
\text { 2. System presents itemized calculated scores per team, round, } \\
\text { damage center, and benefits in the form of a table. }\end{array}$ \\
\hline
\end{tabular}


$\begin{array}{ll}\text { Display } & \text { Admin views } \\ \text { Scores } & \text { summary of } \\ \text { Summary } & \text { scores. }\end{array}$

$\begin{array}{ll}\text { Display } & \text { Admin views } \\ \text { Final } & \text { final scores for } \\ \text { Scores } & \text { rounds and } \\ & \text { tournament. }\end{array}$

1. Admin clicks on Scores Summary tab on the menu.

2. System presents unweighted and custom-weighted scores for expected annual impact, actual impact, and peer scores using hoverable bar charts, along with calculated technical, peer, and total scores for each team.

2.1. Admin interacts with the charts by hovering over a bar.

2.2. System presents the description and value of the bar in a pop-up box.

1. Admin clicks on Final Scores tab on the menu.

2. System presents calculated technical, peer, and total scores per team for each round and tournament total in the form of a table.

2.1. Admin clicks on the title of a column in the table to sort the entries.

2.2. System rearranges the rows with respect to the order of values in the fields of the selected column.

$\begin{array}{ll}\text { Activate } & \text { Admin } \\ \text { Next } & \text { activates the } \\ \text { Round } & \text { second round } \\ & \text { of the } \\ & \text { tournament. }\end{array}$

1. Admin clicks on Dashboard tab on the menu.

2. System displays all teams completed round 1 and enables button to activate round 2 .

3. Admin clicks on the enabled button.

4. System presents a warning dialog for player to confirm irreversible action.

5. Player clicks on confirm button to confirm.

6. System processes the activation and allows players to start round 2. 